\title{
Two-dimensional kinematics of the circumnuclear region of Seyfert galaxies using GMOS-IFU
}

\author{
Fausto K. B. Barbosa ${ }^{1}$, Thaisa Storchi-Bergmann ${ }^{1}$, \\ Claudia Winge ${ }^{2}$, Henrique R. Schmitt ${ }^{3}$ and Roberto Cid Fernandes ${ }^{4}$ \\ ${ }^{1}$ Instituto de Física, UFRGS, C.P. 15001, 91501-970, Porto Alegre, RS, Brazil \\ email: faustokb@if.ufrgs.br \\ ${ }^{2}$ Gemini Observatory, Southern Operations Center, Casilla 603, La Serena, Chile \\ ${ }^{3}$ National Radio Astronomy Observatory, Charlottesville, VA 22903, USA \\ ${ }^{4}$ Departamento de Física-CFM, UFSC, C.P. 476, 88040-900, Florianopolis, SC, Brazil
}

\begin{abstract}
We discuss results of the mapping of both the stellar and gaseous kinematics of the nuclear region of 4 nearby Seyfert galaxies, on the basis of GMOS IFU observations in the spectral region of the CaII triplet $(8500 \AA)$. We do not find in any of these galaxies central drops (within the central hundred parsecs or so) in the stellar velocity dispersions, as recently reported in the literature for a number of Seyferts. However, we do conclude that apparent drops are observed when there is contamination of the CaII triplet absorption lines by underlying line

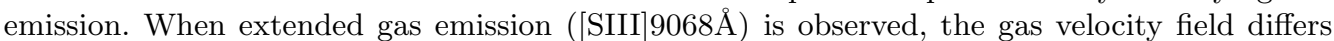
from the stellar velocity field. The latter is usually consistent with the classical "spider diagram" characteristic of disk galaxies.
\end{abstract}

\section{Introduction}

Studies of the gravitational potential of Seyfert galaxies on kiloparsec scales (Nelson \& Whittle 1995) indicate that Seyfert galaxies have lower mean mass-to-light ratios than non-active galaxies, probably due to a younger near-nuclear stellar population (Oliva et al. 1999). More recently, studies of the stellar kinematics at scales of $100 \mathrm{pc}$ have found velocity dispersion drops ( $\sigma$-drops) at the nucleus of some Seyfert galaxies (see contribution of Emsellem to this volume). These drops have been attributed to the presence of a young/intermediate stellar population that does not have the same velocity field as the stars of the bulge. Such an interpretation would be in line with an evolutionary scenario we have proposed on the basis of our recent studies of the stellar population properties around Seyfert nuclei (Schmitt et al. 1999; Cid Fernandes et al. 2001) and their relation with the host galaxy environment (Storchi-Bergmann et al. 2001).

\section{Results}

We have measured the velocity dispersion and radial velocity for the gas and stellar components of 4 Seyfert galaxies: NGC 3227, NGC 3516, NGC 4051 and NGC 4941 using observations with the Integral Field Unit of the Gemini North GMOS spectrograph. The spectral range covered was $8200 \AA-9500 \AA$, to include the Ca II $(\lambda 8500 \AA)$ absorption lines with a spectral resolution of $\simeq 3000$ and spatial sampling of $0.2^{\prime \prime}$. We have measured the stellar velocity dispersion and radial velocities calculating the cross correlation between the galaxy spectra and a standard star spectrum. The gas kinematics was obtained by 

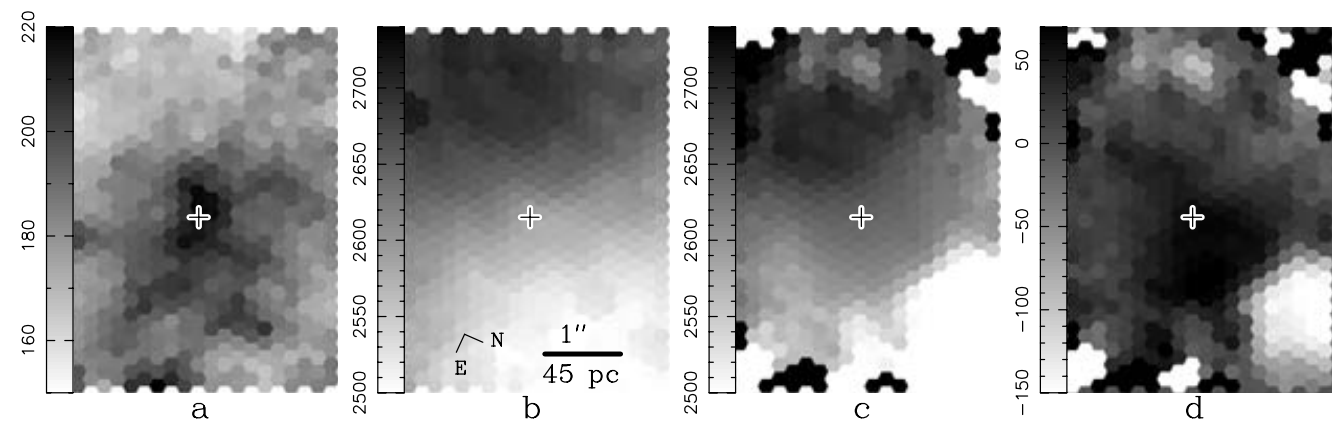

Figure 1. Velocity fields of the inner $3.5^{\prime \prime} \times 5^{\prime \prime}$ region of NGC $3516\left(\mathrm{~km} \mathrm{~s}^{-1}\right)$ : Panel "a": stellar velocity dispersion map; panel "b": stellar radial velocity; panel "c": gas radial velocity; panel "d": difference between the gas and stellar radial velocities. The plus sign marks the maximum of the brightness distribution.

fitting a gaussian function to the [S III] $\lambda 9069$ line and measuring its FWHM and peak wavelength in order to obtain the gas velocity dispersion, radial velocity and flux.

In Fig. 1 we present results for NGC 3516. (Results for the other galaxies will be presented in Barbosa et al. 2004). The stellar velocity dispersion peaks at the nucleus, with an average value of $210 \pm 10 \mathrm{~km} \mathrm{~s}^{-1}$ within the inner $0.6^{\prime \prime}$, consistent with the value listed in Nelson \& Whittle (1995). The stellar radial velocities range from 2490 to $2710 \mathrm{~km} \mathrm{~s}^{-1}$ and approximatedly follow a spider diagram (e.g. Binney \& Merrifield, 1998) typical of disk galaxies. The gaseous velocity field differs considerably from that of the stars, as illustrated in panel "d" of Fig. 1: within 1 arcsec from the nucleus the gas is inflowing, with an average velocity of $60 \mathrm{~km} \mathrm{~s}^{-1}$ relative to the stars, but at 2 $\operatorname{arcsec} \mathrm{N}$ and $2 \operatorname{arcsec} \mathrm{SW}$ from the nucleus, it is outflowing with velocities of -150 and $-100 \mathrm{~km} \mathrm{~s}^{-1}$, respectively, relative to those of the stars.

\section{Conclusions}

We do not find $\sigma$-drops in neither NGC 3516 nor in the other Seyfert galaxies of the sample. An apparent $\sigma$-drop was found in NGC 4051 but which we verified was artificially created by the contamination of the Ca II absorption lines by gas emisison. The stellar velocity field is similar to that expected for disk galaxies (spider diagram). The gaseous velocity field is always different from that of the stars, indicating the presence of kinematic effects not related to the galactic gravitational potential. In the particular case of NGC 3516, within the inner 1 arcsec the gas is inflowing while further out is outflowing relative to the stars.

\section{References}

Barbosa, F. K. B., et al. 2004, in preparation

Binney, J., \& Merrifield, M. 1998, Galactic Astronomy, p.99

Cid Fernandes, R., et al. 2001, ApJ, 558, 81

Emsellem, E. 2004, these Proceedings

Nelson, C. H., \& Whittle, M. 1995, ApJS, 99, 67

Oliva, E., Origlia, L., Maiolino, R., \& Moorwood, A. F. M. 1999, A\&A, 350, 90

Schmitt, H. R., Storchi-Bergmann, T., \& Fernandes, R. C. 1999, MNRAS, 303, 173

Storchi-Bergmann, T., et al. 2001, ApJ, 559, 147 